\title{
Introduction to the special issue on semantic and digital media technologies
}

\author{
Marcin Grzegorzek • Lynda Hardman • David Duce • \\ Siegfried Handschuh • Michela Spagnuolo
}

Published online: 19 January 2010

(C) Springer Science+Business Media, LLC 2010

The objective of this special issue is to collect and report on recent work that aims at narrowing the large disparity between the low-level descriptors that can be computed automatically from multimedia content and the richness of subjectivity of semantics in user queries and human interpretations of audiovisual data. It represents a step towards bridging the gap between research cultures at extreme ends of the semantic multimedia spectrum. At one end, the semantic web and its supporting technologies are becoming established in both the open data environment and within specialist domains, such as corporate intranet search, e-Science (particularly life sciences), and cultural heritage. To facilitate the world-wide sharing of media, W3C is developing standard ways of denoting fragments of audiovisual content and of specifying and associating semantics with these. At the other end of the spectrum, media analysis tools continue to grow in sophistication, identifying features that can then be associated with explicit

M. Grzegorzek $(\bowtie)$

West Institute (Web Science and Technologies), University of Koblenz-Landau, Koblenz, Germany e-mail: marcin@uni-koblenz.de

URL: http://www.uni-koblenz.de/ marcin

L. Hardman

Semantic Media Interfaces Research Group, Centrum voor Wiskunde en Informatica (CWI),

Amsterdam, The Netherlands

e-mail: Lynda.Hardman@cwi.nl

URL: http://homepages.cwi.nl/ lynda

D. Duce

Department of Computing, School of Technology at Oxford Brookes University, Oxford, UK e-mail: daduce@brookes.ac.uk

URL: http://cms.brookes.ac.uk/staff/DavidDuce

S. Handschuh

Digital Enterprise Research Institute, National University of Ireland, Galway, Ireland

e-mail: Siegfried.Handschuh@deri.org

URL: http://www.siegfried-handschuh.net

M. Spagnuolo

Shape Modeling Group, IMATI-Institute for Applied Mathematics and Information Technologies, Genoa, Italy

e-mail: michela.spagnuolo@ge.imati.cnr.it

URL: http://www.ge.imati.cnr.it/ima/spagnuolo.htm 
semantics expressed formally or informally, using proprietary formats or open standards. Recent progress at these two fronts of the spectrum means that research spanning the semantic gap is now of vital importance to feed the real applications that are emerging.

In this issue of Multimedia Tools and Applications one may find eleven contributions:

"A Framework for Video Abstraction Systems Analysis and Modelling from an Operational Point of View" by Victor Valdes and Jose M Martinez presents a unified taxonomy and a generic architectural model aimed for the study of existing abstraction systems computational performance and characteristics.

"A Semantic Management Model to enable the integrated management of media and devices" by Paula Viana and Artur Pimenta Alves proposes a new ontology that aggregates information from different sources and enables a top level business oriented view of multimedia archives.

"Automatic Summarization of Rushes Video using Bipartite Graphs" by Alan F. Smeaton, Liang Bai, Songyang Lao, and Noel E O'Connor presents a new approach for automatic summarization of rushes, or unstructured video.

"Automatic Tag Expansion using Visual Similarity for Photo Sharing Websites" by Sare Gul Sevil, Onur Kucuktunc, Pinar Duygulu, and Fazli Can describes an automatic photo tag expansion method designed for photo sharing websites.

"Enlightening the Sun: A User Interface to Explore Music Artists via Multimedia Content" by Markus Schedl and Tim Pohle proposes an approach to browse collections of web pages about music artists by means of descriptive terms and multimedia content. "Everyday Concept Detection in Visual Lifelogs: Validation, Relationships and Trends" by Daragh Byrne, Aiden R Doherty, Cees G. M Snoek, Gareth J. F Jones, and Alan F. Smeaton explores the applicability of semantic concept detection, a method often used within video retrieval, on the domain of visual lifelogs.

"GameSense: Game-like In-Image Advertising" by Lusong Li, Tao Mei, and XianSheng Hua presents a novel game-like advertising system called GameSense, which is driven by the compelling contents of online images.

"Investigating Fuzzy DLs-Based Reasoning in Semantic Image Analysis" by Stamatia Dasiopoulou, Ioannis Kompatsiaris, and Michael G. Strintzis investigates a fuzzy DLsbased reasoning framework, which enables the integration of scene and object classifications into a semantically consistent interpretation by capturing and utilising the underlying semantic associations.

"Performing Content-based Retrieval of Humans using Gait Biometrics" by Sina Samangooei and Mark S Nixon explores the content-based retrieval of videos containing walking subjects, using semantic queries. It evaluates current research in gait biometrics, unique in its effectiveness at recognising people at a distance.

"Semantic-driven Multimedia Retrieval with the MPEG Query Format" by Ruben Tous and Jaime Delgado describes extensions which will allow MPQF to manage metadata modelled with Semantic Web languages like RDF and OWL, and query constructs based on SPARQL. The suggested modifications include the definition of a new MPQF query type, and a generalization of the MPQF metadata processing model. "Thesaurus Enrichment for Query Expansion in Audiovisual Archives" by Laura Hollink, Veronique Malaise, and Guus Schreiber aims at finding out whether retrieval of audiovisual resources from a collection indexed with an in-house thesaurus can be improved by enriching the thesaurus structure. It proposes propose a method to add structure to a thesaurus by anchoring it to an external, semantically richer thesaurus. 


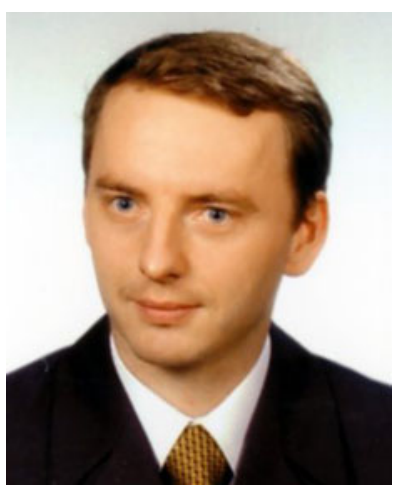

Marcin Grzegorzek is a Lecturer in the Institute for Web Science and Technologies (WeST) at the University of Koblenz-Landau (Germany), where he leads the Multimedia Web (MMWeb) Focus Group. He graduated in Computer Science at the Silesian University of Technology in Gliwice (Poland) in 2002. In 2007 he received his $\mathrm{PhD}$ (summa cum laude) in the field of statistical object recognition from the University of Erlangen-Nuremberg (Germany). Moreover, he worked as a Postdoctoral Research Assistant for the Multimedia and Vision Research Group at the Queen Mary University of London (UK). Marcin Grzegorzek presented his scientific results on international conferences and workshops, in international journals, as well as in form of a text book. He acts as a Secretary in the Executive Board of the SMaRT (Semantic Multimedia Research and Technology) Association. Moreover, he is a Founding Committee Member of the Institute for Medicine Engineering and Information Processing (MTI Mittelrhein), an interdisciplinary facility bringing together researchers from the Mittelrhein area who work on new solutions for medical applications. His current research topics belong to the fields of image processing, pattern recognition, and multimedia retrieval.

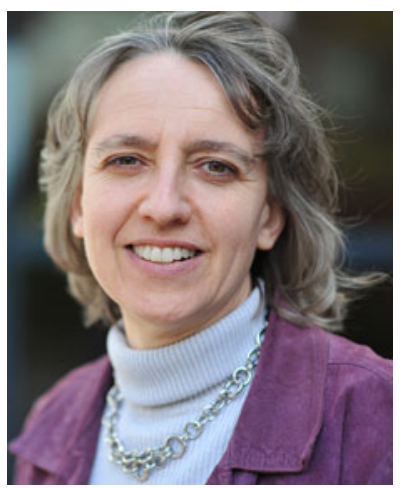

Lynda Hardman is head of the Interactive Information Access group at CWI (Centrum Wiskunde \& Informatica) and professor by special appointment of Multimedia Interaction in the Faculty of Science at the University of Amsterdam. She obtained her PhD from the University of Amsterdam in 1998, having graduated in Mathematics and Physics from Glasgow University in 1982. During several years of working in the software industry she was the development manager for Guide — the first hypertext authoring system for personal computers (1986). Her early experiences in industry with the development of hypertext authoring tools, before the Web as we know it developed, inspired her towards underlying questions of combining time-dependent documents (such as video sequences) along with interaction through links into a single model. She was a member of the $\mathrm{W} 3 \mathrm{C}$ working group that developed the first SMIL recommendation. Since the development of the semantic web, she has dedicated herself to improving human access to the ever-expanding "linked data cloud". Her current research efforts are focused on improving design methods for human-based interfaces in relation to developing technology. She is a member of the editorial board for the Journal of Web Semantics, and the New Review of Hypermedia and Multimedia, and was co-programme chair for SAMT 2008. 


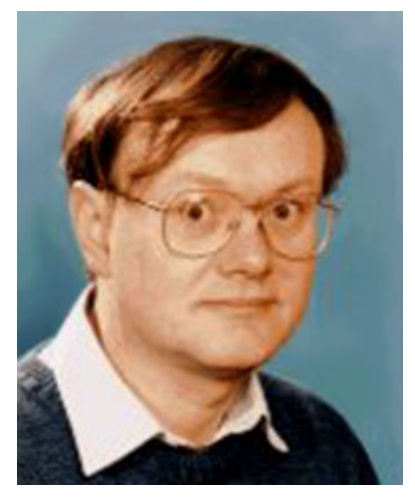

David Duce is a Professor in Computer Science in the School of Technology at Oxford Brookes University, where he leads the Web Technologies research group. He has been involved in computer graphics since 1975. He was one of the editors of the ISO/IEC standard for computer graphics, the Graphical Kernel System (GKS), and has co-author of books on GKS, PHIGS and Graphics Standards. He was also involved in the development of the Computer Graphics Reference Model and Presentation Environments for Multi-media Objects (PREMO). Most recently he was co-editor for Portable Network Graphics (PNG) second edition, which is a W3C Recommendation and an ISO/IEC standard (ISO/IEC 15948:2004). His main current research interests are in visualisation, distributed systems, Web graphics and applications of Semantic Web technology. He is currently Vice-Chairman of the Eurographics Association, treasurer of the International World Wide Web Conference Steering Group (IW3C2), and a member of the UK Computing Research Committee (UKCRC. He is on the Editorial Board of the journal Formal Aspects of Computing.

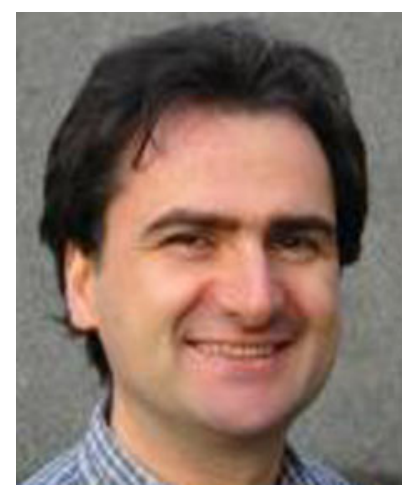

Dr. Siegfried Handschuh is a SFI Stokes Senior Lecturer at the National University of Ireland, Galway (NUIG). $\mathrm{He}$ is the research leader of the Semantic Collaboration research stream at the Digital Enterprise Research Institute (DERI). Siegfried holds Honours Degrees in both Computer Science and Information Science and a PhD from the University of Karlsruhe. He published over 90 papers as books and journal, book chapters, conference, and workshop contributions, mainly in the areas of Annotation and Authoring for the Semantic Web, Knowledge Acquisition, Information Visualisation and Social Semantic Collaboration. His publication record includes prestigious journal and conferences such as Computer Networks, IEEE Transactions, Journal of Web Semantics, ACM SIGIR, WWW, ISWC and ESWC. He is well cited, with an h-index over 20. Since 2000 he has initiated, participated and/or coordinated several R\&D projects at an international level, such as DAML (DARPA), aceMedia (IP), HALO 2 (Vulcan Inc.), Knowledge Web (NOE) and Nepomuk (IP). 


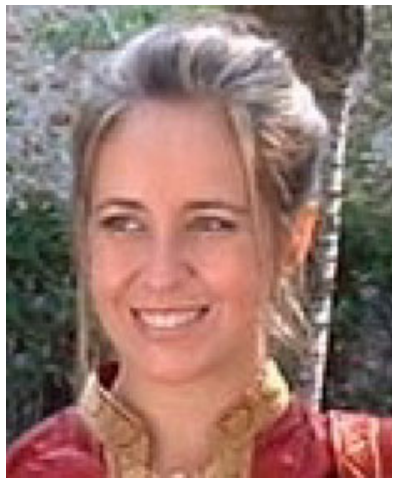

Michela Spagnuolo is a Senior Researcher at IMATI-CNR, where she is a member of the Shape Modeling Group. She received a Laurea in Mathematics from the University of Genova, Italy, and the Doctorate degree in Computer Science Engineering from the INSA Lyon, France. Her research interests are related to computational topology for 3D shape analysis and understanding, classification and retrieval, semanticsdriven methods for modelling and processing digital shapes. She has authored more than 130 publications, and has been program chair of IEEE Shape Modeling International (SMI), Semantic and Digital Media Technology (SAMT) and EG workshops of 3D Object Retrieval (3DOR). 